\title{
ON-LINE SEVERITY ASSESSMENT OF BEARING DAMAGE VIA DEFECT SENSITIVE RESONANCE IDENTIFICATION AND MATCHED FILTERING
}

\author{
C. JAMES LI \\ Department of Mechanical Engineering, Columbia University, New York, NY 10027, U.S.A.
}

AND

S. M. WU

Department of Mechanical Engineering and Applied Mechanics, University of Michigan, Ann Arbor, MI 48109, U.S.A.

(Received August 1987, accepted February 1988)

\begin{abstract}
A microcomputer based on-line bearing condition monitoring system was developed. Employing synchronised segmentation and parametric spectral comparisons, the system enabled on-line identification of defect sensitive resonances for an investigated bearing system at an early stage of damage. A matched filter was designed to keep track of the energy contributed by these resonances throughout the rest of bearing life. The magnitude of the energy was found to be well correlated with the development of bearing localised defects. It takes $38 \mathrm{sec}$ for the identification of defect sensitive resonances and $7 \mathrm{sec}$ for the matched filter to report each new assessment of bearing condition on a programmed PC-AT.
\end{abstract}

\section{INTRODUCTION}

Within industry the ability to assess the extent of bearing damage has many uses. When the extent of bearing damage is taken into account with other factors such as production schedule, spares availability etc., an optimal decision may be made when the bearing should be changed. Some signal processing techniques [1]-[8] were developed to detect the onset of bearing defects based on the identification of characteristic defect frequencies (Appendix A) or the observation about the changes from the normal signals. However, neither the characteristic defect frequencies nor the pattern of the changes will consistently reflect the severity of damage throughout the entire process of bearing damage [1].

The passing of a rolling element upon a localised defect generates a wide band impulse. Particular resonances in the bearing system and nearby structure are excited more by the impulses resulting from damage than by normal operation of the machine or other sources of excitation. They are denoted as "defect sensitive resonances". In a bearing system, either the enlargement of defects or the increase in the number of them will result in the increase of the vibration energy contributed by defect sensitive resonances.

This paper presents a newly developed scheme for the assessment of the severity of bearing damage and an on-line implementation of the scheme. Employing synchronised segmentation and parametric spectral comparisons, the scheme enables on-line identification of defect sensitive resonances for a given bearing system without having recourse to the bearing's historical data. Using the defect sensitive resonances as a template, a matched filter designed to watch the variations of energy contributed by the defect sensitive resonances is used to assess the condition of the bearing throughout the rest of its life. 
During normal operation, the vibration of a bearing system is monitored by the pattern classification technique to detect the onset of bearing localised defects [9]. The detection of the onset of defects will trigger the synchronised segmentation on the signal of bearing. The synchronised segmentation will generate two subsequences between which defect sensitive resonances contribute different amounts of energy. Thus, defect sensitive resonances may be identified by the template learning machine which compares the difference in the energy of each resonance between these two subsequences.

Bearings with simulated defects are monitored for changes in signals due to the development of damage. To provide a comparison, some of the state-of-the-art techniques and the proposed scheme are applied to assess the extent of the damage. Their effectiveness and efficiency are evaluated and compared.

\section{SYNCHRONISED SEGMENTATION}

It was found that one usually has no easy way of knowing the resonances of a given bearing system, much less to say which of these are going to be excited by impulses generated by a damaged bearing [1]. One can either guess the band in which such a resonance will be or wait until a bearing gets damaged to identify the exact frequency of such a resonance by comparing signals measured at different stages of damage.

Neither of the two approaches will be useful for on-line applications for the following reasons:

a. The guess may turn out to be wrong. There is no guarantee of a correct guess.

b. Before one can identify any defect sensitive resonance through the comparisons of bearing signals, the bearing has to be damaged at least once, which is a very significant limitation.

c. Because signals have to be measured at different stages of bearing damage, it is possible that some of the differences between them will have nothing to do with the damage of the bearing.

In order to enable the on-line identification of defect sensitive resonances, a technique denoted as "synchronised segmentation" and a template learning machine were developed to identify defect sensitive resonances in a "single shot" manner. The segmentation technique is able to generate two subsequences from a single piece of signal measured at a bearing's early stage of damage. Simply comparing these two subsequences by using the template learning machine will reveal the defect sensitive resonances. Thus, the identification of defect sensitive resonances may be carried out without having recourse to historical records of bearing signals.

At the initial stage of bearing damage, an impulse train at one of the characteristic defect frequencies excites the bearing system's resonances to generate a series of regularly spaced vibration bursts (Fig. 1(a)). A sequence starting at the beginning of any one of the bursts ending right before the beginning of the next one is denoted as a "burst sequence" (Fig. 1(a)).

The synchronised segmentation technique works as follows. After the detection of the onset of defects, every vibration burst is highlighted by the short-time signal processing techniques (Appendix B). Note how the peaks in the short-time energy function reflect the vibration bursts in the bearing signal (Fig. 1(b)). Because damage related bursts should occur at the rate of one of the characteristic defect frequencies, a spurious burst may be easily rejected if it does not occur at the right moment. Then, every defect related burst sequence is segmented out of the bearing signal and cut into two halves. The first halves of these burst sequences are combined into subsequence one. Subsequence two is 

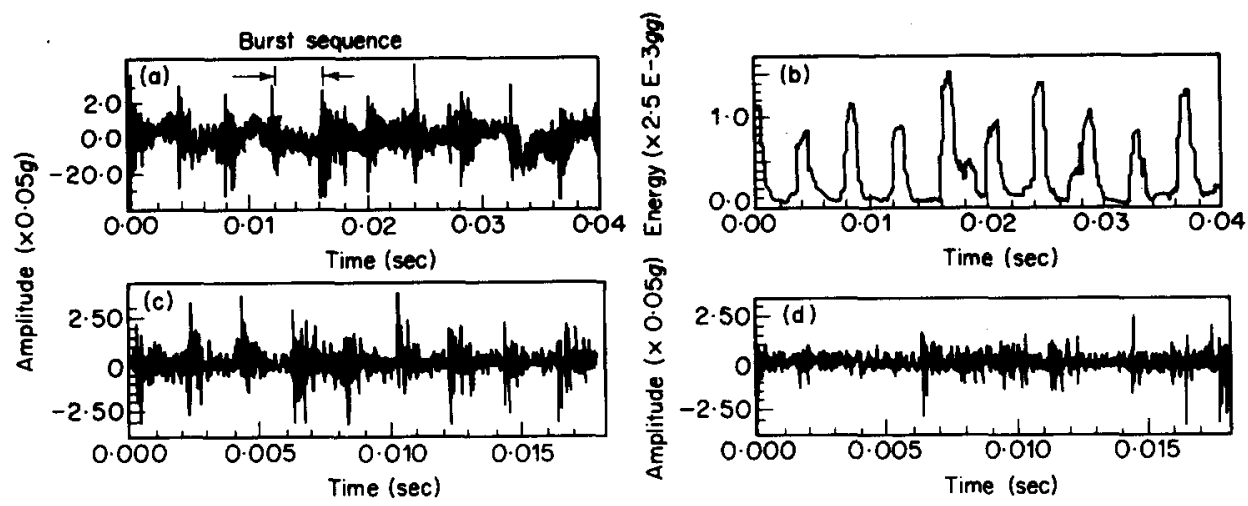

Figure 1. Subsequence formation by synchronised segmentation. (a) The bearing vibration. (b) Short-time energy function. (c) The first subsequence. (d) The second subsequence.

formed in a similar manner from the second halves of the burst sequences. Figures $1(\mathrm{c})$ and 1 (d) are subsequences formed from the bearing signal displayed in Fig. 1(a).

It is evident that a defect sensitive resonance will have a larger amplitude at the beginning of a burst sequence than at the end of it. Therefore, vibrational energy contributed by any defect sensitive resonance in subsequence one will be significantly higher than that in subsequence two.

\section{IDENTIFICATION FOR DEFECT SENSITIVE RESONANCES VIA A TEMPLATE LEARNING MACHINE}

Defect sensitive resonances are identified in the following way. First, every recognisable resonance in both subsequences is identified. Among them, a resonance contributing significantly more energy to subsequence one than to subsequence two is regarded as a defect sensitive resonance. A template learning machine employing Dynamic Data System (DDS) methodology was designed to carry out the above stated process. The DDS methodology is outlined first.

\subsection{DYNAMIC DATA SYSTEM METHODOLOGY (DDS)}

The DDS is a modeling technique which uses the operating data in the form of a time series to develop difference/differential equations [10]. If a physical system under investigation can be approximately represented by a linear differential equation, then it is possible to fit a difference equation, called an Autoregressive Moving Average (ARMA) model, to the uniformly sampled operating data from the system. For an $n$th order continuous model of the system, it can be mathematically shown that there is an $\operatorname{ARMA}(n, n-1)$ model in discrete form as

$$
X_{t}-\phi_{1} X_{t-1}-\cdots-\phi_{n} x_{t-n}=a_{t}-\theta_{1} a_{t-1}-\cdots-\theta_{n-1} a_{t-n+1}
$$

where $X_{t}$ is the time series, $\theta_{t}$ are the moving average parameters, $a_{t}$ are the residuals with $\operatorname{NID}\left(0, \sigma_{a}^{2}\right)$ and $\phi_{t}$ are the autoregressive parameters.

To estimate the modal parameters, $\phi_{i}$ 's and $\theta_{t}$ 's from a given series of observations, a sequential modeling strategy is employed. It involves fitting models of higher order using the method of non-linear least squares which minimises the sum of squares of the residuals, $a_{i}$, until a satisfactorily adequate model is obtained. A detailed illustration of this procedure is given in ref. [10]. The adequate model contains pertinent information 
available from the system. The system's frequency spectrum can be estimated from the model's parameters by the transformation as

$$
f(\omega)=\frac{\sigma_{a}^{2}}{2 \pi} \frac{\left|\mathrm{e}^{(n-1) i \omega}-\theta_{1} \mathrm{e}^{(n-2) i \omega}-\theta_{2} \mathrm{e}^{(n-2) i \omega}-\cdots-\theta_{n-1}\right|^{2}}{\left|\mathrm{e}^{n i \omega}-\phi_{1} \mathrm{e}^{(n-1) i \omega}-\phi_{2} \mathrm{e}^{(n-2) i \omega}-\cdots-\phi_{n}\right|^{2}}
$$

where $\omega$ is the frequency in radians.

The fitted model $\operatorname{ARMA}(n, n-1)$ has its eigen-equation

$$
\lambda^{n}-\sum_{j=1}^{n} \phi_{j} \lambda^{n-j}=0
$$

and

$$
\nu^{n-1}-\sum_{j=1}^{n-1} \theta_{j} \nu^{n-1-j}=0
$$

where $\lambda$ and $\nu$ are characteristic roots of the autoregressive part and the moving average part respectively.

The complex conjugate characteristic roots $\left(\lambda, \lambda^{*}\right)$ represent the dynamic models generated by vibration sources of the underlying system. The total energy of the vibration signal can be expressed by

$$
\begin{gathered}
\operatorname{Var}\left(x_{t}\right)=\gamma_{0} \\
\gamma_{0}=\sum_{i=1}^{n} \sum_{j=1}^{n} g_{i} g_{j} \sigma^{2} \frac{1}{1-\lambda_{i} \lambda_{j}} \\
g_{j}=\prod_{k=1}^{n-1}\left(1-\nu_{k} \lambda_{j}^{-1}\right) / \prod_{k=1(k \neq j)}^{n}\left(1-\lambda_{k} \lambda_{j}^{-1}\right) .
\end{gathered}
$$

Each term in equation (6) represents the contribution due to the mode $\left(\lambda_{i}, \lambda_{i}^{*}\right)$ to the total energy. The percentage, in which each mode contributes to $\gamma_{0}$, is called dispersion $P_{i}$,

$$
P_{i}=\frac{\sum_{j=1}^{n} g_{i} g_{j} \sigma_{a}^{2} \frac{1}{1-\lambda_{i} \lambda_{j}}}{\gamma_{0}} 100 \%
$$

The damping ratio $\zeta$ and natural frequency $f_{n}$ of the corresponding mode can be obtained as

$$
\begin{gathered}
\zeta=\ln \left(\lambda \lambda^{*}\right) / \sqrt{\left[\ln \left(\lambda \lambda^{*}\right)\right]^{2}+4\left[\cos ^{-1}\left(\frac{\lambda+\lambda^{*}}{2 \sqrt{\lambda \lambda^{*}}}\right)\right]^{2}} \\
f_{n}=\frac{\pi}{\Delta} \sqrt{\left[\ln \left(\lambda \lambda^{*}\right)\right]^{2}+4\left[\cos ^{-1}\left(\frac{\lambda+\lambda^{*}}{2 \sqrt{\lambda \lambda^{*}}}\right)\right]^{2}} .
\end{gathered}
$$

The S-plane characteristic root pair of this mode may be easily computed from the relationship: $\mu \mu^{*}=\omega_{n}^{2}$ and $\mu+\mu^{*}=-2 \zeta \omega_{n}^{2}$.

To summarise, the natural frequency, damping ratio and energy dispersion of each underlying system's resonance and a parametric spectrum of the operation data can be developed directly from the operational data through the use of DDS methodology.

\subsection{TEMPLATE LEARNING MACHINE}

The schematic block diagram of the learning machine is shown in Fig. 2. Subsequences one and two are processed independently by DDS methodology so that every resonance 


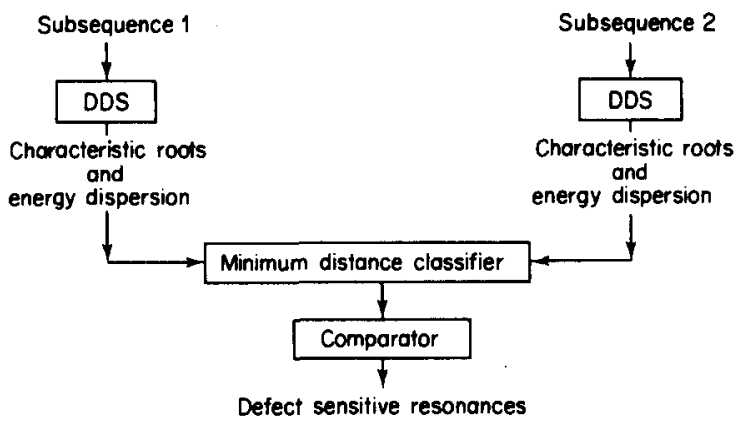

Figure 2. Block diagram of template learning machine.

as well as its energy dispersion are estimated. Thus, one will obtain one set of roots and their corresponding energy from each subsequence.

The minimum distance classifier identifies two pairs of roots that represent the same system mode from the first and second set of roots respectively. The difference of the energy dispersion of this mode between two subsequences is computed by the comparator. Resonances with large energy differences are regarded as the defect sensitive resonances.

\subsection{MINIMUM DISTANCE CLASSIFIER}

Representing the same system mode, two pairs of roots estimated from two subsequences respectively will not have identical values. This is due to the inexactness of the ARMA parameter estimation procedure. Given two sets of roots, one will have to make stochastic decisions about which two pairs of roots are most likely from the same system's mode. A minimum distance classifier employing Bayes' decision theory was designed to minimise the probability of making a wrong decision.

To facilitate the following illustration, a hypothetical distribution of characteristic roots is given in Fig. 3. Since the distribution is always symmetric to the real axis, only the upper half plane including the real axis is shown. Clearly, only one characteristic root will appear in this portion of the S-plane for each resonance. Roots estimated from subsequence two are indicated by circles and labeled in lowercase characters. Roots estimated from subsequence one are indicated by square boxes and labeled by numbers. Energy dispersion values are given near each root.

The two categories Bayes' decision rule for minimising the probability of decision error is:

$$
\text { Decide } \omega_{1} \text { if } P\left(\omega_{1} \mid \mathbf{y}\right)>P\left(\omega_{2} \mid \mathbf{y}\right) \text {; otherwise decide } \omega_{2} \text {. }
$$

where $y$ represents the observed feature vector and $\omega=\left\{\omega_{1}, \omega_{2}\right\}$ represents the set of

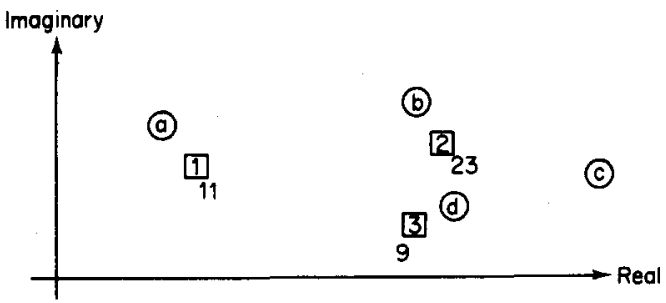

Figure 3. The distribution of roots in a complex plane. 
possible categories. According to the Bayes rule:

$$
P\left(\omega_{j} \mid \mathbf{y}\right)=\frac{p\left(\mathbf{y} \mid \omega_{j}\right) P\left(\omega_{j}\right)}{p(\mathbf{y})}
$$

where

$$
p(\mathbf{y})=\sum_{j=1}^{c} p\left(\mathbf{y} \mid \omega_{j}\right) P\left(\omega_{j}\right) .
$$

An equivalent decision rule by applying the Bayes rule will be:

Decide $\omega_{1}$ if $p\left(\mathbf{y} \mid \omega_{1}\right) P\left(\omega_{1}\right)>p\left(\mathbf{y} \mid \omega_{2}\right) P\left(\omega_{2}\right)$; otherwise decide $\omega_{2}$.

A discriminant function derived from this theory is defined as:

$$
d_{i}(\mathbf{y})=\log \left[p\left(\mathbf{y} \mid \omega_{i}\right) P\left(\omega_{i}\right)\right] .
$$

Then, the Bayes decision rule becomes:

Decide class $i$ if $d_{i}>d_{j}$.

Now, if one assumes:

(a) densities $p\left(y \mid \omega_{i}\right)$ are of multivariate normal distribution. Let

$$
p\left(\mathbf{y} \mid \omega_{i}\right) \sim N\left(\boldsymbol{\mu}_{i}, \mathbf{\Sigma}_{i}\right)
$$

where $\boldsymbol{\mu}$ is the distribution mean vector and $\boldsymbol{\Sigma}$ is the distribution covariance matrix.

(b) each component in the feature vector $y$ is independent, and each has the same variance $\left(\Sigma=\sigma^{2} I\right.$, where $I$ is the identity matrix $)$, and

(c) priori probabilities $P\left(\omega_{i}\right)$ are the same for all classes.

Then the discriminant function becomes:

$$
d_{i}(\mathbf{y})=\frac{\left\|\mathbf{y}-\boldsymbol{\mu}_{i}\right\|}{2 \sigma^{2}}
$$

where $\|\cdot\|$ is the Euclidean norm.

In this case, the optimum decision rule can be stated as follows: To classify a feature vector $\mathbf{y}$ into one of the $n$ categories, one measures the Euclidean distance $\left\|\mathbf{y}-\boldsymbol{\mu}_{\mathrm{i}}\right\|$ from $y$ to each of the $n$ mean vectors, and then classifies to the category of the nearest mean. Such a classifier is called a minimum distance classifier.

The above derived relationship, equation (14) is applied in a straightforward manner. The feature vector, $y$, is the co-ordinate pair of the real and imaginary part of a characteristic root. The set of possible categories for a root in the first set of characteristic roots is the complete second set of characteristic roots. Thus, the discriminant function is the distance measured in the S-plane from this root to any root in the second set.

The procedure of root classifying is illustrated by using Fig. 3. Since a resonance with higher energy dispersion should be given priority, the root classifying is performed in descending order of energy dispersion. Starting with the root which has the largest energy dispersion value in the first set of characteristic roots (root 2 in this case), the minimum distance classifier classifies it into the nearest root (root b) in the second set. Then, both of them are dropped from both sets to keep them from being involved in further classification procedure. The same procedure continues with the remaining roots until all the roots in the first set are successfully classified. 


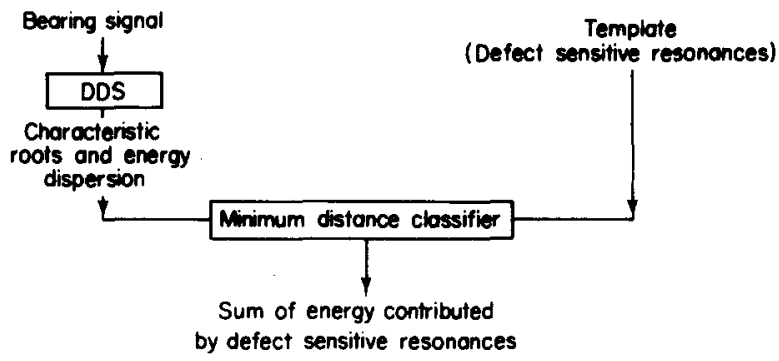

Figure 4. Block diagram of matched filter.

\section{DAMAGE SEVERITY ASSESSMENT VIA MATCHED FILTERING}

Once the defect sensitive resonances are identified, total energy contributed by them is a good indication about the extent of damage. For the purpose of estimating the energy contributed by defect sensitive resonances is bearing signals, an efficient matched filter technique is used based on the known defect sensitive resonances.

The block diagram of the matched filter is presented in Fig. 4. The defect sensitive resonances are input from a template data file in the form of characteristic roots and corresponding energy dispersion values. An adequate model of the incoming bearing signal is estimated by DDS methodology. The S-domain mapping of model's AR characteristic roots and their energy dispersion are computed. Among these characteristic roots, the minimum distance classifier considers the one with the shortest distance from a defect sensitive resonance to be generated by the same vibration mode behind that defect sensitive resonance.

The vibrational energy associated with such a root is calculated by multiplying its energy dispersion by the square root of the bearing signal's variance. The sum of energy contributed by all of them is used as a severity index of the bearing damage.

\section{ON-LINE BEARING CONDITION MONITORING SYSTEM}

\subsection{SYSTEM ORGANISATION}

In the on-line implementation, there are a data acquisition stage and a data processing stage. The data acquisition stage includes four pieces of hardware: (a) an accelerometer, (b) a charge amplifier, (c) a band pass filter, and (d) an analog to digital (A/D) converter. Also, there are four functional units in the data processing stage: (a) the defect detection/diagnosis unit, (b) the unit of synchronised segmentation, (c) the template learning machine, and (d) the matched filter. The defect detection/diagnosis unit is responsible for detecting the onset of localised defects [9]. The other three units have been discussed in sections 2, 3 and 4 of this paper respectively. Figure 5 is the block diagram which illustrates the organisation of the system.

In the center of the system, a supervisor is responsible for the proper logic sequence of system operation, the control of the data flow between each functional unit and the global data base, and the management of the global data base.

The use of a global data base implemented on the mass storage equipment of the computer system facilitates the exchange of information among the data acquisition stage, the four functional units of the data processing stage, and the system's interface. There are two more advantages for using such a data base, namely (a) not all of the data have to reside in the computer's memory simultaneously, (b) the data base can be used to 


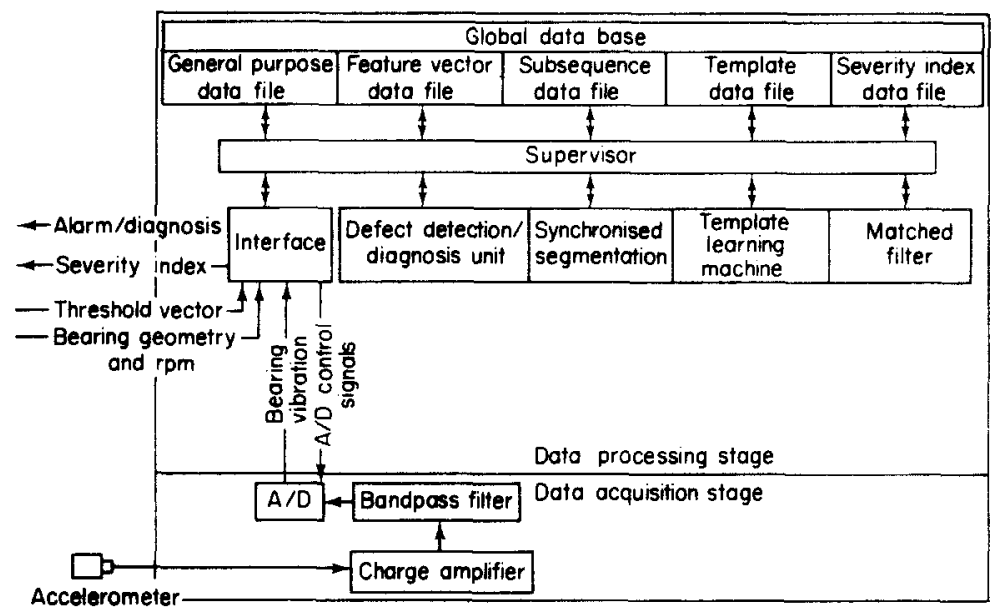

Figure 5. Block diagram representation of on-line bearing monitoring system.

preserve short-term data prior to the shutdown of the monitored system or monitoring system itself.

\subsection{SYSTEM OPERATION}

Upon receiving the necessary information from a human operator (e.g. bearing geometry), the supervisor activates the A/D converter. The data supplied by the A/D converter are formulated into records and written on to a general purpose data file.

The data are first processed by the defect detection/diagnosis unit. The process of data measuring and processing will be continuously carried out until the detection and diagnosis of any localised defect. In the case of such an event, a warning message will be displayed on the CRT to alert the operator. Following this, two subsequences are generated by synchronised segmentation and stored in the subsequence data files.

With these on-line generated subsequences, the template learning machine identifies defect sensitive resonances and writes them into a template data file. Thus, the defect sensitive resonances become available for the matched filter.

The matched filter then keeps track of the vibration energy contributed by defect sensitive resonances and outputs it as the severity index for bearing damage periodically.

\section{EXPERIMENTAL SET-UP AND RESULTS}

\subsection{EXPERIMENTAL SET-UP}

The schematic diagram of the experimental set-up which consists of a spindle driven by an $\mathrm{AC}$ motor and a mechanical platform is shown in Fig. 6. The mechanical platform

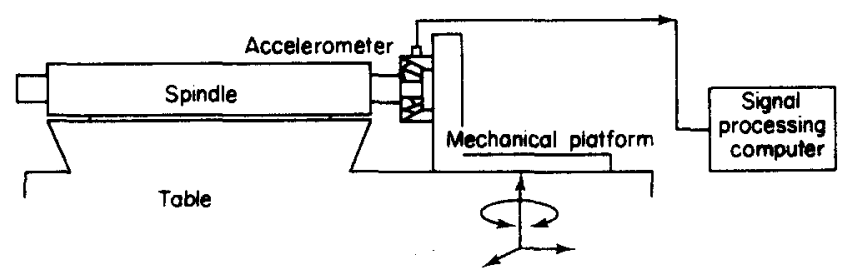

Figure 6. Schematic diagram of experimental set-up. 
enables the adjustment for the following: the thrust load, the angular misalignment, and the position of the center of the bearing. The cup and cone of a taper roller bearing under test are mounted on the platform and the spindle respectively. The number and location of defects imposed on the tested bearings by an electrical discharge machine included the following: (1) single outer-race defect, (2) single roller defect, (3) both single roller and outer-race defect, (4) three outer-race defects, and (5) six outer-race defects. The size of the defects is 60/1000 in. in diameter by 5/1000 in. in depth which is typical [11]. General purpose grease was applied to each of the bearings for lubrication.

A PCB 303A02 accelerometer was mounted on the outer surface of the bearing housing to pick up its vibrations in the radial direction. These vibrations are bandpass filtered (5-25k) and digitised before they are sent to the signal processing computer (a PC-AT).

\subsection{RESULTS OF DEFECT SENSITIVE RESONANCE IDENTIFICATION}

The ability to identify defect sensitive resonances of a given bearing system was tested by experiments carried out on the experimental set-up using a bearing with an outer-race defect and a bearing with a roller defect.

AR(20) models were used to implement the template learning machine. Table 1(a) and 1(b) list the three strongest resonances found in the signals. The defect sensitive resonance is assumed to be the resonance that has the largest difference, $P 1-P 2$, in energy dispersion value between two subsequences ( $P 1$ and $P 2$ denote the energy dispersion of the resonance estimated from subsequences 1 and 2 respectively). It can be seen from Table 1(a) that no matter how the rotational speed or the load were varied, the resonance at approximately $21.5 \mathrm{kHz}$ was consistently identified as the defect sensitive resonance by the template learning machine for the outer-race damaged bearing. Similarly, the resonance at $10 \cdot 5 \mathrm{kHz}$ was identified as the defect sensitive resonance for the roller damaged bearing (Table 1(b)).

\subsection{DATA PROCESSING RESULTS OF MATCHED FILTERING}

The matched filter was designed to compute the energy (not energy dispersion) contributed by defect sensitive resonances in bearing signals. Bearing signals can be considered to consist of two components: one related to defect sensitive resonances, the desired "signal", and the other from all other sources, the unwanted "noise". Using the template (a defect sensitive resonance) extracted by the template learning machine, the matched filter recovers the "signal" component from bearing signals and estimates the vibration energy contributed by it.

The output of the matched filter, the energy contributed by the defect sensitive resonance in the vibration of damaged bearings, is plotted against the number of outer-race defects in Fig. 7(a). The energy is estimated by multiplying the square root of the sum of square of a vibration sequence and the dispersion, $P$, of the resonance. We adopted the absolute energy instead of the dimensionless energy dispersion, $P$, because the dispersion of a defect sensitive resonance may be affected by the change of the strength of other resonances that have nothing to do with the damage. It is the trend of the absolute energy contributed by a defect sensitive resonance which reflects the condition of a bearing.

Three different levels of thrust load were applied to all the test bearings. Each curve presents the trend of the vibration energy contributed by the defect sensitive resonance under one of the loading conditions. Regardless of the loading conditions, all the curves indicate monotonic up-hill trends as the number of outer-race defects increases.

In contrast to the matched filtering scheme, the trends displayed by state-of-the-art techniques like peak to peak, variance, crest factor, kurtosis and HFRT techniques are not able to fully reflect the real condition of the bearing (Fig. 7(b)-(f)). Generally speaking, peak to peak values or variance values of bandpassed bearing signals do increase as the 
TABLE 1

Defect sensitive resonance identified by the template learning machine

(a) Outer-race damaged bearing

\begin{tabular}{|c|c|c|c|c|c|c|c|}
\hline & $\mathrm{r} / \mathrm{min}$ & $\begin{array}{l}\text { Thrust } \\
\text { load }\end{array}$ & $\begin{array}{l}\text { Natural } \\
\text { frequency } \\
(\mathrm{Hz})\end{array}$ & $\begin{array}{c}P 1 \\
(\%)\end{array}$ & $\begin{array}{c}P 2 \\
(\%)\end{array}$ & $\begin{array}{c}P 1-P 2 \\
(\%)\end{array}$ & $\begin{array}{l}\text { Natural } \\
\text { frequency } \\
(\mathrm{Hz})\end{array}$ \\
\hline 1 & 2038 & Small & 21236 & $24 \cdot 36$ & $11 \cdot 25$ & $13 \cdot 11$ & 18545 \\
\hline 2 & 2038 & Moderate & 21489 & $20 \cdot 28$ & $6 \cdot 42$ & $13 \cdot 86$ & 18583 \\
\hline 3 & 1804 & Moderate & 21579 & $29 \cdot 15$ & $7 \cdot 9$ & $21 \cdot 25$ & 18480 \\
\hline 4 & 2030 & Large & 21794 & $17 \cdot 36$ & $9 \cdot 44$ & $7 \cdot 92$ & 18501 \\
\hline \multirow[t]{2}{*}{5} & 2172 & Large & 21211 & $14 \cdot 38$ & $1 \cdot 74$ & $12 \cdot 64$ & 18336 \\
\hline & $\begin{array}{c}P 1 \\
(\%)\end{array}$ & $\begin{array}{c}P 2 \\
(\%)\end{array}$ & $\begin{array}{c}P 1-P 2 \\
(\%)\end{array}$ & $\begin{array}{c}\text { Natural } \\
\text { frequency } \\
(\mathrm{Hz})\end{array}$ & $\begin{array}{c}P 1 \\
(\%)\end{array}$ & $\begin{array}{c}P 2 \\
(\%)\end{array}$ & $\begin{array}{c}P 1-P 2 \\
(\%)\end{array}$ \\
\hline i & $17 \cdot 33$ & $16 \cdot 72$ & 0.61 & 10300 & $21 \cdot 64$ & $16 \cdot 24$ & $5 \cdot 40$ \\
\hline 2 & $29 \cdot 21$ & $27 \cdot 7$ & $1 \cdot 51$ & 10235 & $14 \cdot 52$ & $11 \cdot 0$ & $3 \cdot 52$ \\
\hline 3 & $31 \cdot 62$ & $35 \cdot 38$ & $-3 \cdot 76$ & 10277 & $7 \cdot 88$ & $19 \cdot 41$ & $-11 \cdot 53$ \\
\hline 4 & $26 \cdot 01$ & $20 \cdot 34$ & 5.67 & 10610 & $6 \cdot 12$ & $12 \cdot 98$ & -6.86 \\
\hline 5 & $5 \cdot 15$ & $3 \cdot 4$ & 1.75 & 10046 & $7 \cdot 51$ & $5 \cdot 51$ & $2 \cdot 00$ \\
\hline
\end{tabular}

(b) Roller damaged bearing

\begin{tabular}{|c|c|c|c|c|c|c|c|}
\hline & $\mathrm{r} / \mathrm{min}$ & $\begin{array}{l}\text { Thrust } \\
\text { load }\end{array}$ & $\begin{array}{c}\text { Natural } \\
\text { frequency } \\
(\mathrm{Hz})\end{array}$ & $\begin{array}{c}P 1 \\
(\%)\end{array}$ & $\begin{array}{c}P 2 \\
(\%)\end{array}$ & $\begin{array}{c}P 1-P 2 \\
(\%)\end{array}$ & $\begin{array}{c}\text { Natural } \\
\text { frequency } \\
\quad(\mathrm{Hz})\end{array}$ \\
\hline 1 & 2032 & Small & 21546 & $15 \cdot 26$ & $7 \cdot 53$ & $7 \cdot 73$ & 18284 \\
\hline 2 & 2032 & Moderate & 21518 & $8 \cdot 18$ & 8.04 & $0 \cdot 14$ & 18431 \\
\hline \multirow[t]{2}{*}{3} & 2032 & Large & 21949 & $12 \cdot 01$ & $10 \cdot 60$ & $1 \cdot 41$ & 18420 \\
\hline & $\begin{array}{c}P 1 \\
(\%)\end{array}$ & $\begin{array}{c}P 2 \\
(\%)\end{array}$ & $\begin{array}{c}P 1-P 2 \\
(\%)\end{array}$ & $\begin{array}{c}\text { Natural } \\
\text { frequency } \\
(\mathrm{Hz})\end{array}$ & $\begin{array}{c}P 1 \\
(\%)\end{array}$ & $\begin{array}{c}P 2 \\
(\%)\end{array}$ & $\begin{array}{c}P 1-P 2 \\
(\%)\end{array}$ \\
\hline 1 & $20 \cdot 57$ & $28 \cdot 91$ & $-8 \cdot 34$ & 10217 & $31 \cdot 86$ & $8 \cdot 99$ & $22 \cdot 87$ \\
\hline 2 & $10 \cdot 45$ & $9 \cdot 27$ & $1 \cdot 18$ & 10523 & $22 \cdot 60$ & $12 \cdot 16$ & $10 \cdot 44$ \\
\hline 3 & $25 \cdot 6$ & $25 \cdot 2$ & 0.4 & 10404 & $21 \cdot 5$ & $13 \cdot 19$ & $8 \cdot 31$ \\
\hline
\end{tabular}

number of defects increases. However, neither of them was monotonic under all three loading conditions. Both crest factor and kurtosis performed very poorly in reflecting the extent of damage. The performance of HFRT is better than the crest factor analysis and the kurtosis analysis but worse than the variance and peak to peak value.

Due to the complexity of the non-linear least square algorithm required by the DDS model estimation, fixed ordered AR approximation was used instead of the $\operatorname{ARMA}(n, n-$ 1) model in the on-line implementation of the proposed scheme. The approximation simplifies the implementation of both template learning machine and matched filter and reduces a great deal of data processing time. However, the best possible performance of the matched filter and the template learning machine was sacrificed.

Both the matched filter and the template learning machine were implemented using AR(20) models. In order to probe the effect of this approximation, we carry out matched 


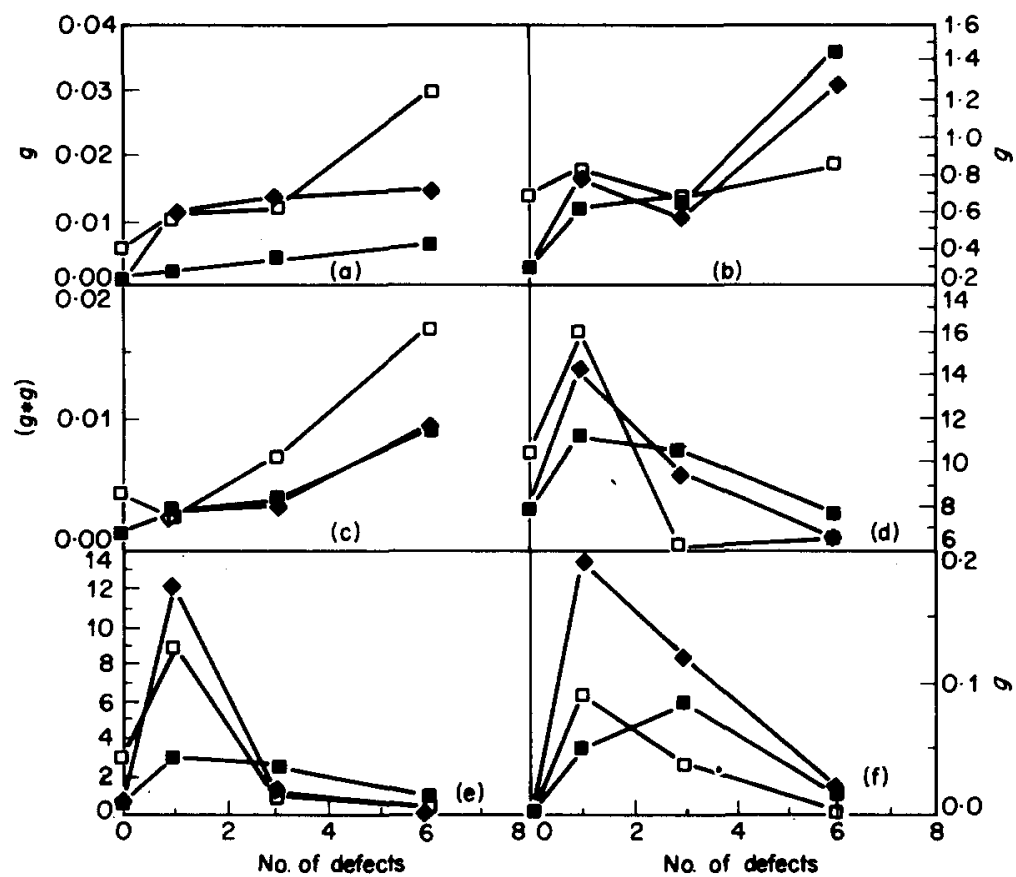

Figure 7. Damage severity index. (a) Matched filtering. (b) Peak to peak. (c) Variance. (d) Crest factor. (e) Kurtosis. (f) HFRT. $\square$, Small thrust loads; $\bullet$, moderate thrust loads; $\square$, large thrust loads.

filtering on a signal to which increasing amounts of random noise are added. The benchmark used was the simulated response of a single degree-of-freedom system subjected to white noise excitation obscured by random noise. The natural frequency and damping ratio of the system are $50 \mathrm{rad} / \mathrm{sec}$ and $0 \cdot 01$.

Some quantitative results of the matched filter acting on the benchmark signals are shown in Table 2 . In all cases, $\operatorname{ARMA}(4,3)$ were found to be adequate for modeling the contaminated signals. Generally speaking, the signal can be recovered with good accuracy before the signal to noise $(\mathrm{S} / \mathrm{N})$ ratio is dropped to $-20 \mathrm{~dB}$. However, the lower the $\mathrm{S} / \mathrm{N}$ ratio the bigger the error of signal recovery. Note that the error in the estimation of damping ratio is more sensitive to the noise contamination. The result of using $\operatorname{AR}(4)$ models in the matched filter acting on the benchmark signals are also shown in Table 2. The amount of the degradation is noticeable.

TABLE 2

The effect of $S / N$ ratio upon matched filtering

\begin{tabular}{ccccc}
\hline & \multicolumn{4}{c}{ Matched filtering } \\
\cline { 2 - 5 } $\begin{array}{c}\text { Signal/noise } \\
\text { ratio(dB) }\end{array}$ & \multicolumn{2}{c}{ ARMA(4, 3) } & \multicolumn{3}{c}{ AR(4) approximation } \\
\cline { 2 - 5 } & $\omega(\mathrm{rad} / \mathrm{sec})$ & $\zeta$ & $\omega(\mathrm{rad} / \mathrm{sec})$ & $\zeta$ \\
\hline$\infty$ & 49.98 & 0.0083 & 49.61 & 0.0099 \\
1 & 50.78 & 0.015 & 48.49 & 0.083 \\
-10 & 50.39 & 0.0274 & 47.99 & 0.2 \\
-20 & 47.81 & 0.37 & 51.25 & 0.44 \\
\hline
\end{tabular}

The natural frequency and damping ratio of the system are $50 \mathrm{rad} / \mathrm{sec}$ and 0.01 . 
The on-line implementation of the ARMA model estimation will become feasible as emerging high performance microprocessors and computer algorithms specially tailored for the DDS models become available. Once this is achieved, the condition of the bearing will be assessed on-line more precisely by the matched filter. If a matched filter is expected to recover signals at even lower $\mathrm{S} / \mathrm{N}$ ratio (e.g. $<-20 \mathrm{~dB}$ ), one will have to employ more advanced modeling strategies such as non-linear DDS[12].

\section{CONCLUSIONS}

1. A self-learning scheme for in-process identification of defect sensitive resonances was developed to identify on-line the system dependent defect sensitive resonances for a bearing system. This scheme is (a) able to identify the bearing defect sensitive resonances without human intervention, and (b) does not need any historic records of the investigated bearing system.

2. The matched filter has proven to be an effective on-line bearing condition assessing scheme. Compared with the other existing bearing condition monitoring techniques, the matched filter (a) gives a clearly increasing trend as the number of defects increases, and (b) may be used for recovering signals buried in noise.

3. The performance of both the template learning machine and the matched filter can be improved further once the on-line ARMA modeling become available.

4. The designed template learning machine and matched filter can also be applied to any computerised signal processing system that is required to carry out spectrum comparisons and template matching.

5. An on-line bearing condition monitoring system was integrated based upon the developed schemes.

\section{ACKNOWLEDGMENT}

Support from General Motors is gratefully acknowledged.

\section{REFERENCES}

1. P. D. MCFADDEN and J. D. SMITH 1984 Tribology International January, 3-10. Vibration monitoring of rolling element bearings by the high frequency resonance technique-a review.

2. E. DoWNHAN and R. WoodS, ASME Paper No. 71-Vibr-96. The rationale of monitoring vibration on rotating machinery in continuously operating process plant.

3. B. WEICHBRODT and J. BOWDEN March 1970 Report S-70-1021 AD 869633, GE Co. instrument for predicting bearing damage.

4. D. DYER and R. M. STEWART 1978 Journal of Mechanical Design 100, 229-235. Detection of rolling element bearing damage by statistical vibration analysis.

5. O. G. Gustafsson and T. TAllian 1962 Transactions ASLE 5, No. 62, 197-209. Detection of damage in assembled rolling bearings.

6. F. F. EHRICH 1972 Journal of Engineering for Industry 181-184. Sum and difference frequencies in vibration of high speed rotating machinery.

7. R. L. Eshleman 1980 Proceedings of the Machinery Vibration Seminar, IV, Cherry Hill, $N J$, Vibration Institute 1-18. The use of sum and difference frequencies in rotating machinery analysis.

8. S. BRAUN and B. DATner 1979 Journal of Mechanical Design 101, 118-125. Analysis of roller/ball bearing vibrations.

9. C. LI 1987 ASME Symposium on Intelligent and Integrated Manufacturing Analysis and Synthesis PED 25, 141-152. On-line bearing localized defects detection by pattern recognition analysis.

10. S: M. WU ASME Transactions Series B 99, 708-714. Dynamic data system-a new modeling technique. 
11. R. L. WIDNER and W. E. LITTMANN 1976 National Bureau of Standard Special Publication No. 423. Bearing damage analysis.

12. Y. T. SU $1986 \mathrm{Ph} . \mathrm{D}$ dissertation University of Wisconsin, Madison, WI. Nonlinear time series model development and application in control.

13. J. M. BAKER 1975 Ph.D dissertation, Carnegie-Mellon University, Pittsburgh, PA. A new time-domain analysis of human speech and other complex waveforms.

14. J. B. Allen 1977 IEEE Transactions, Acoustics, Speech and Signal Processing, 25, 235-238. Short-term spectral analysis and synthesis and modification by discrete Fourier transform.

15. L. D. ERMAN 1975 Ph.D. dissertation, Carnegie-Mellon University, Pittsburgh, PA. An environment and system for machine understanding of connected speech.

\section{APPENDIX A: CHARACTERISTIC DEFECT FREQUENCIES}

During the bearing operation, impulses are generated from the rolling element-race way contacts. At the initial stage of bearing damage, each defect in the main bearing elements (inner-race, roller or outer-race) has a characteristic frequency at which impulses are generated $\left(f_{i r}, f_{r o}\right.$ and $\left.f_{o r}\right)$. They are denoted as characteristic defect frequencies.

$$
\begin{gathered}
f_{o r}=\frac{n}{2} f_{r}\left(1-\frac{B D}{P D} \cos (\beta)\right) \\
f_{i r}=\frac{n}{2} f_{r}\left(1+\frac{B D}{P D} \cos (\beta)\right) \\
f_{\text {ro }}=\frac{B D}{P D} f_{r}\left(1+\left(\frac{P D}{B D}\right)^{2} \cos (\beta)\right)
\end{gathered}
$$

where $f_{o r}$ is the characteristic defect frequency for defect on outer-race; $f_{i r}$ is the characteristic defect frequency for defect on inner-race; $f_{r o}$ is the characteristic defect frequency for defect on roller; $B D$ is the roller diameter; $P D$ is the bearing pitch diameter; $f_{r}$ is the bearing rotating speed; $\beta$ is the contact angle between race and ball.

\section{APPENDIX B: SHORT-TIME SIGNAL PROCESSING TECHNIQUES}

There are a variety of "short-time" processing methods in which short segments of a bearing signal are isolated and processed as if they were short segments from a sustained bearing vibration signal with fixed properties. This is repeated periodically as often as desired. Such processing produces a new time-dependent sequence which can serve as a representation of the vibration signal.

The short-time energy function of a signal is defined as

$$
E_{n}=\sum_{m=-\infty}^{\infty} x^{2}(m) w(n-m)
$$

where $w(n)=1$ if $0 \leqslant n \leqslant N-1,=0$ otherwise. Other short-time signal processing techniques include short-time average zero crossing rate, median smoothing, short-time Fourier analysis, and short-time autocorrelation [13]-[15]. 